\title{
RICE DISEASE IDENTIFICATION AND CLASSIFICATION BASED ON MATLAB AND ANDROID PLATFORM
}

\author{
Zhou Hui ${ }^{1}$, Zhang Tianpeng ${ }^{2}$, Lin Hong ${ }^{1}$, Xiao Maohua ${ }^{1}$, Wu Zijian ${ }^{1}$, Wu Juanjuan ${ }^{1}$ \\ ${ }^{1}$ College of Engineering, Nanjing Agricultural University, Nanjing, 210031,China \\ ${ }^{2}$ Kunshan Agricultural Mechanization Technology Extension Station, Suzhou, 215300, China \\ Email:xiaomaohua@njau.edu.cn
}

\begin{abstract}
To achieve rapid and portable automatic identification and classification of rice diseases, this paper proposes texture feature value comparison, the multiple linear regression equation, the BP (back propagation) neural network three-step recognition method using the log-sigmoid function, and the convolutional neural network (CNN) comprehensive recognition method. First, the background, the leaves, and the lesion areas are thresholded according to the color histogram. After morphological processing, the texture, morphological, and color features are extracted and used as the input parameters of the three-step recognition model. The third step constructs a $3 \times 6$ $\times 2$ three-layer BP neural network prediction model with the tan-sigmoid function as the hidden layer neuron activation function to identify the remaining similar diseases and finally transplants the algorithm to the mobile phone Android platform. The comparative analysis of the visual experiments reveals that the proposed method has an average recognition accuracy of $95.5 \%$ for the five types of diseases, an average recognition accuracy increase of $3.1 \%$, and an average classification accuracy of $88.9 \%$ compared with direct classification using only CNNs. This research on rice disease identification can provide reference on multiple types of disease identification and disease degree classification.
\end{abstract}

Keywords: Rice Diseases; Identification and Classifications; MachineVisions; Image Processings; Mobile Application.

\section{Introduction}

China is a big country in rice cultivation and consumption, and the stable production of rice is of great significance to its food security. Rice blast is an important rice disease. Serious rice blast can result in a $40 \%-50 \%$ reduction in or even no grain yield. Rice blast is mainly prevented and controlled by spraying pesticides ${ }^{[1]}$, which often does not follow a control standard, and different grades and types of disease have different pesticide requirements.

Computer vision technology has played a pivotal role in the intelligent diagnosis of crop diseases, especially leaf diseases ${ }^{[2]}$. Therefore, a computer can identify the type and extent of rice disease and then determine the pesticide spraying method that can greatly reduce the cost of manpower and material resources and environmental pollution. People are increasingly using machine vision technology to replace human eyes in agricultural production with the rapid development of computer technology, enabling the step by step establishment of a rice disease identification grading model through multiple logistic regression equations and machine vision technology.

Some scholars have performed automatic equipment identification and analysis on crops, such as wheat[3], corn[4-5], tomato[6], and cotton[7], especially the extraction of diseased area images. Image segmentation is a technique and process of dividing an image into regions with various characteristics and extracting the target of interest[8]. In a previous research, the stepwise discriminant analysis method was used to select the characteristic parameters of each disease feature ${ }^{[9]}$, and the screening and classification of the disease spots focused on image processing by adding color, shape, texture, and other conditions layer by layer. Some methods only use deep convolutional neural networks (CNNs) to identify diseases. The general classification accuracy is between $90 \%$ and $93 \%$ when the number of disease classifications is $3-8^{[10-}$ 11]. The correct recognition rate obtained by our group is only $93.3 \%$ when using only CNNs, which is basically similar with the results of other scholars, but the recognition rate increases by $3.1 \%$ after adding the re-verification method. 
The addition of the revalidation group has a positive effect on the improvement of the recognition accuracy.

\section{Material Acquisition and Processing}

\subsection{Obtaining Rice Disease Spots}

Diseased intact rice leaves were obtained from rice fields in Nanjing in August 2019. The obtained leaves were placed on flat A4 white papers, a smartphone was fixed on a tripod, and a mobile phone camera was placed on the ground. A total of 100 valid pictures were obtained for each type of disease, that is, 500 pictures in 5 groups. And query the rice disease atlas ${ }^{[12]}$ or database to obtain ${ }^{[13]}$. The image preprocessing software uses MATLAB2018a, which was introduced by Mathworks. The processing equipment is a $\mathrm{PC}$, and the basic configuration CPU is as follows: i5, 8G memory, and 256G hard disk.

\subsection{Image Preprocessing}

For convenient image preprocessing and unified image specifications, the acquired image is cropped into a $4000 \times 3000$ geometric image. The acquired image is a 32-bit true color image.

Consequently, a large amount of data is handled during image transmission and processing. Jones ${ }^{[14]}$ and Oliver ${ }^{[15]}$ found that the image color space can be converted to $\mathrm{Y}, \mathrm{Cb}$, and $\mathrm{Cr}$ space images for the threshold segmentation of cluster-like pictures.
$\mathrm{Cb}$ represents the blue density deviation of the color, and $\mathrm{Cr}$ represents the red density deviation of the color. The slight deviation between $\mathrm{Cb}$ and $\mathrm{Cr}$ is nearly imperceptible to humans, so we can use $\mathrm{Y}, \mathrm{Cb}$, and $\mathrm{Cr}$ for sub-sampling to compress the image data.

First, the pre-processed R, G, and B images are converted into the $\mathrm{Y}, \mathrm{Cb}$, and $\mathrm{Cr}$ space.

$$
\left\{\begin{array}{l}
Y=0.299 R+0.587 G+0.114 B \\
C_{b}=-0.172 R-0.339 G+0.511 B+128 \\
C_{r}=0.511 R-0.428 G-0.083 B+128
\end{array}\right.
$$

where R-_R channel gray value,

$G--G$ channel gray value,

$B-$ - $B$ channel gray value.

The standard color histogram under the $\mathrm{Y}, \mathrm{Cb}$, and $\mathrm{Cr}$ color space is obtained, the gray value of the $\mathrm{R}, \mathrm{G}, \mathrm{B}$ channels in this color space is measured using the Takecolor 8.0 software, and the gray value of the background and other noise is measured via Range exclusion for a clearer image threshold cutting range. The color feature-based positioning algorithm is based on intuitive color features ${ }^{[16]}$, and the standard color histogram is obtained by Formula (2) ${ }^{[17]}$.

$$
h_{C_{I}}=\operatorname{Pr}\left[P \in I_{C_{I}}\right]=\frac{H_{C_{I}}}{w \times h}
$$

HCI(I)-_-Histogram of image I

$\mathrm{P}$-_Pixels in the image

$\mathrm{w} \times \mathrm{h}-$ - Image size
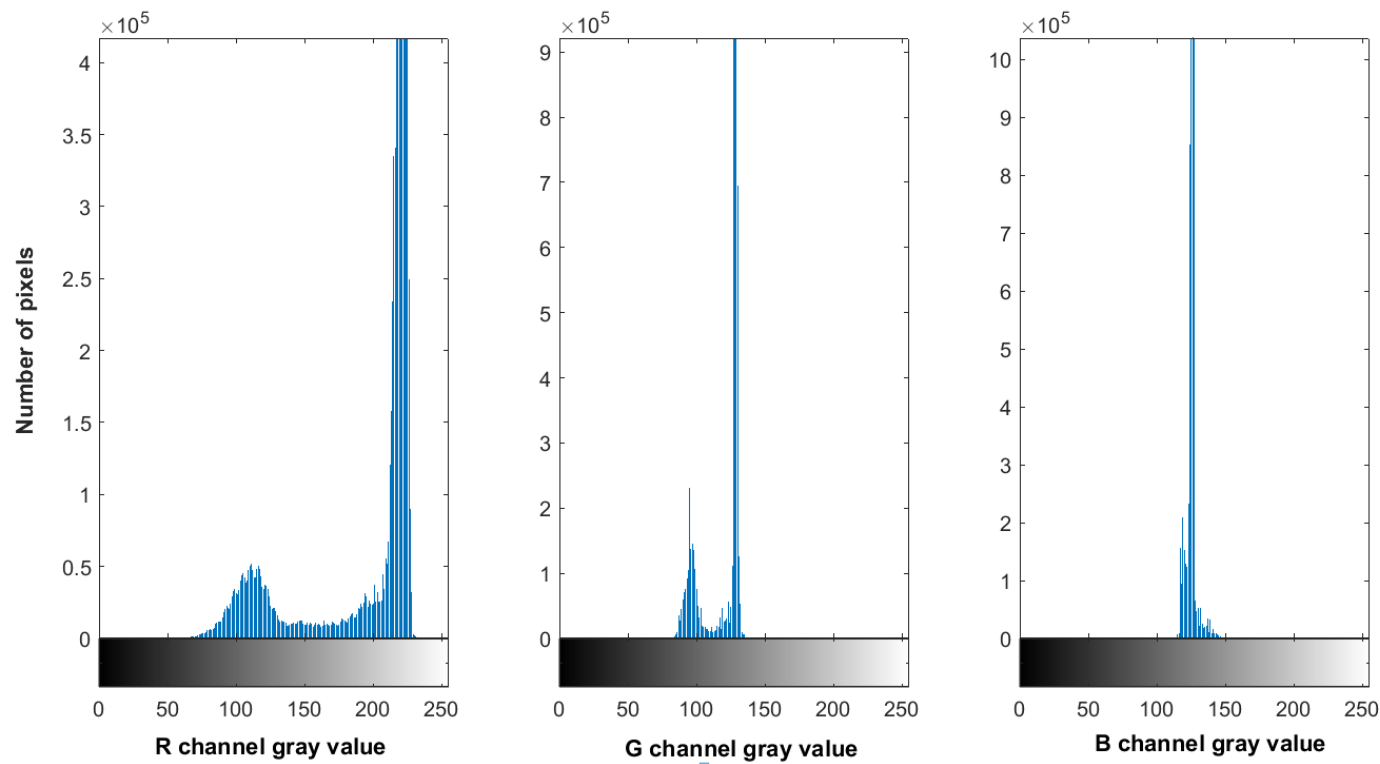

Figure 1: R, G, B three-channel color histogram

According to the color distribution histogram, the Takecolor 8.0 software is used to sample the color gray value for the subsequent threshold segmentation. 
Table 1 Three-channel threshold segmentation of

\begin{tabular}{cc}
$\begin{array}{c}\text { Threshold } \\
\text { segmentation }\end{array}$ & $\begin{array}{c}\text { Gray value segmentation } \\
\text { range }\end{array}$ \\
\hline $\mathrm{R}$ & $40-210$ \\
$\mathrm{G}$ & $105-125$ \\
$\mathrm{~B}$ & $135-140$ \\
\hline
\end{tabular}

Takecolor 8.0 sampling indicates that the background color is mostly concentrated in the range of the gray value above 210 in the $\mathrm{R}$ channel. This part is directly cut and eliminated.

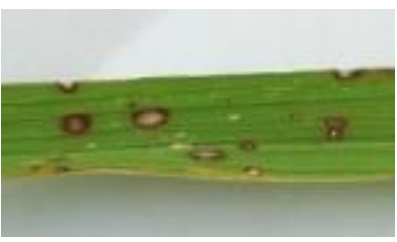

A

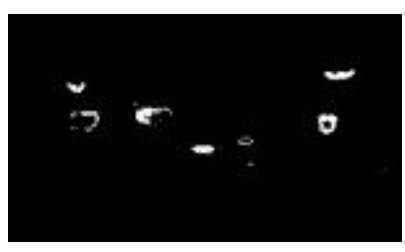

E

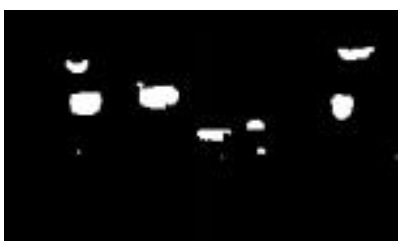

I

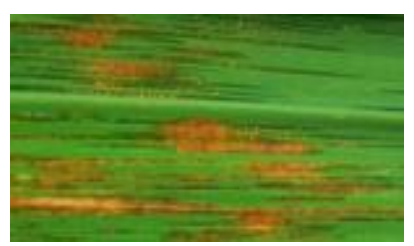

B

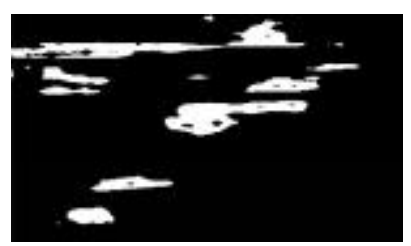

F

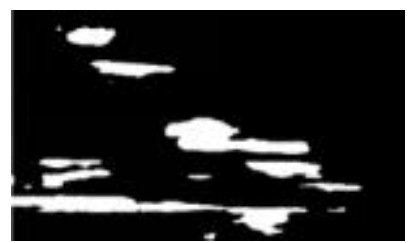

$\mathrm{J}$
The target area of leaves and stems that must be directly segmented is concentrated in the peak area of the $\mathrm{G}$ channel. The histogram of the lesion area shows the peak area in the B channel.

The center of the lesion area of the binarized image after segmentation is not detected, and a part of the circle has defects and cannot be used as the final calculation data of the lesion area. The open operation (corrosion first and swelling) separates objects at slender points and smoothens the boundaries of larger objects without changing their area significantly, filling the blank area in the center of the diseased spot. Finally, the infill function is called.

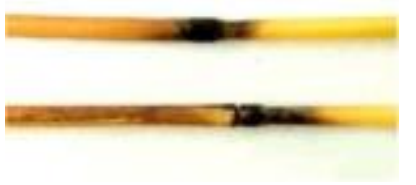

C

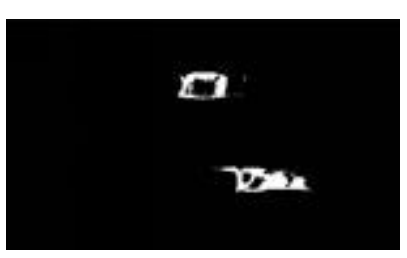

G

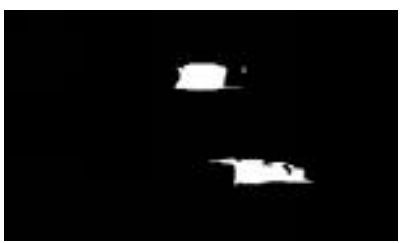

$\mathrm{K}$

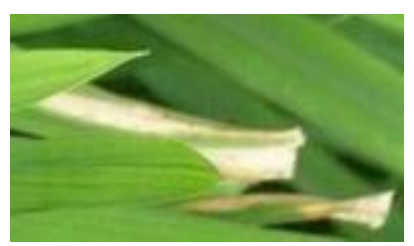

D

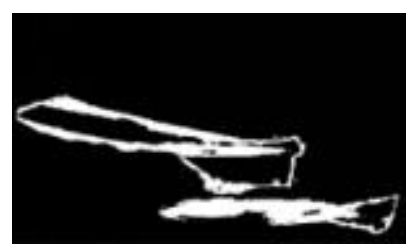

$\mathrm{H}$

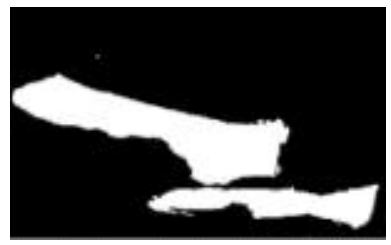

L

A. Chronic leaf blast; B. Bacterial stripe; C. Arthritis; D. Rice leaf tip blight; E. Binarization of chronic leaf blast; F. Binarization of bacterial stripe disease; G. Binarization of arthritis; $H$. Binarization of rice leaf tip blight; $I$. Morphological treatment of chronic leaf blast; J. Morphological treatment of bacterial stripe; K. Morphological treatment of arthritis; L. Morphological treatment of rice leaf tip blight

Figure 2: Morphological processing of lesion image

\subsection{Morphological Feature Extraction}

There are 5 types of disease images. After the disease image is preprocessed, the color, texture, and morphological features in the image are extracted, and the disease is identified by gradually adding feature values to screen the specified disease ${ }^{[18]}$. Formulas (3)-(4) are respectively used to obtain the aspect ratio and eccentricity of the lesion.

$$
r=\frac{W_{M E R}}{L_{M E R}}
$$

$$
E=\sqrt{\frac{(A+B)-\sqrt{(A+B)^{2}+4 H^{2}}}{(A+B)+\sqrt{(A+B)^{2}+4 H^{2}}}}
$$

r-C The ratio of the width to the length of the smallest bounding rectangle

WMER-—The width of the smallest bounding rectangle

LMER- - The length of the smallest bounding rectangle

A, B, C--Moment of inertia of a rigid body around the $\mathrm{x}, \mathrm{y}, \mathrm{z}$ coordinate axis

$\mathrm{H}-$-Product of inertia 
Table 2 Rice disease shape, color and texture

\begin{tabular}{c}
\hline Disease name \\
\hline Rice blast (chronic) \\
Rice bacterial leaf streak \\
Rice leaf tip blight \\
Arthritis
\end{tabular}

\subsection{Lesion area measurement}

In the $\mathrm{YCbCr}$ color space, the gray value of the color channel where the central gray-white area is located is judged through the color distribution histogram, and the area is obtained by a separate segmentation. The area of the remaining lesions is the number of pixels occupied by the target.

$$
A=\sum_{x=0}^{m-1} \sum_{y=0}^{n-1} \int(x, y)
$$

\begin{tabular}{cc}
\multicolumn{2}{c}{ Feature name } \\
Shape & Color and texture \\
Elliptic or fusiform & Centre: Ashen; Edge: Brown \\
Slim strip & Dark brown or yellow \\
Strip & white-grey, edges: brown \\
Dotted or nearly round & Black or brown
\end{tabular}

m--Image rows

n--Number of image columns

$f(x, y)-$ The pixel value of the $x$ th row and $y$ th column of the image

\section{Disease Identification}

\subsection{Disease Identification Process Design}

In this study, the stepwise discriminant analysis method is used to select the characteristic parameters of each disease feature ${ }^{[19-22] .}$

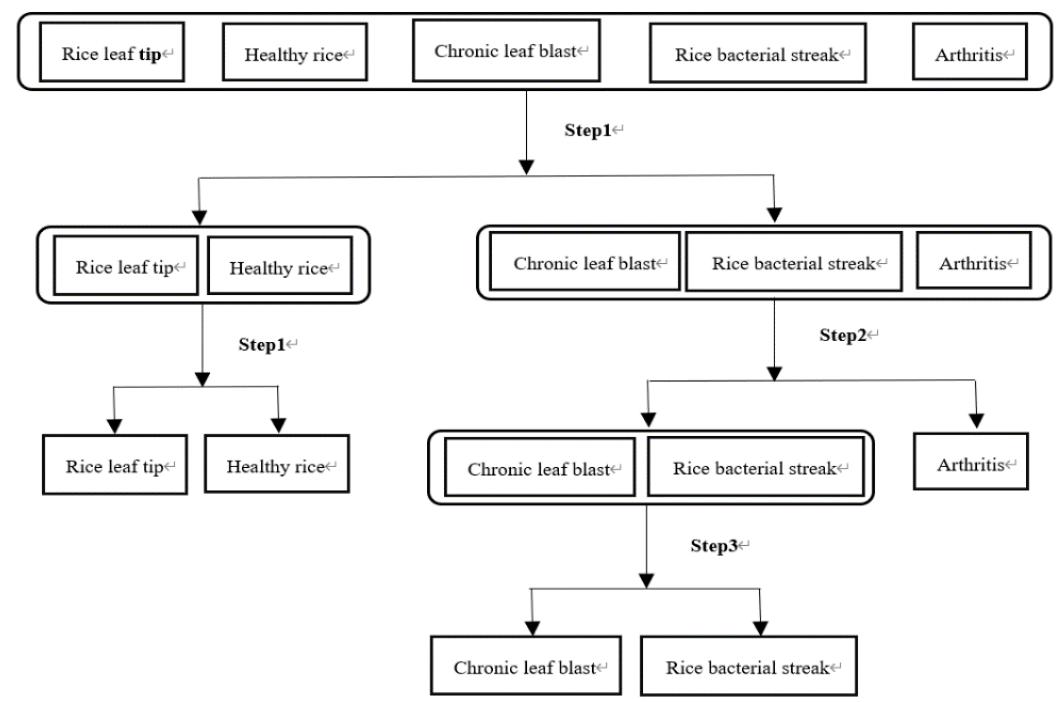

Figure 3: Flow chart of rice disease classification

Step 1 screens the image by the ratio of the graywhite lesion area in the center of the lesion to the entire lesion area in the texture feature and initially divides the disease types into three groups. Step 2 performs multiple fitting by adding morphological feature values, establishes multiple regression equations, and divides the remaining three types into knots and other groups. Finally, chronic leaf blast and bacterial leaf streak were screened. Step 3 adds the color feature condition based on the shape feature value and uses the BP neural network to predict and analyze the two disease types.

\subsection{Step1 Texture Features}

The analysis of the ratio of the central area of the lesion to the entire area of the lesion indicates that most of the lesion areas are not composed of a single color. The center of leaf tip blight and chronic leaf blast is gray-white and surrounded by an brown oval ring. This leaf tip blight feature is particularly prominent. The ratio of the central gray area to the entire diseased area is usually $0.95-0.99$. In healthy rice, no disease spots can be detected and directly judged. The ratio of the remaining three types of lesion images is generally below 0.6 and has no obvious correlation. Thus, the three types cannot be directly identified and judged by this feature.

\subsection{Step2 Morphological Characteristics}

On the basis of the texture features, shape features are added again, namely, aspect ratio and eccentricity. The number of disease spots on a single leaf obtained after segmenting the disease image by thresholding is different. Dozens of connected 
domains of diseased spots will appear in the severely diseased rice leaves. The average value of the characteristic values of disease spots on a single leaf was extracted for analysis.

The morphological characteristic values of the 195 rice diseases classified into 3 groups of 65 diseases each were measured and extracted. Then, 25 and 40 are taken from each group as the test and verification sets, respectively. Finally, 195 sets of eccentricity and aspect ratio data were substituted for binary linear fitting, and a binary regression prediction model was established.
After obtaining the binary linear regression equation, the proposed verification group of aspect ratio and eccentricity is substituted into the equation to verify the accuracy of fitting, where $R$ is the regression value of the three diseases.

$$
R=0.4015-0.9282 * A+0.5214 * E
$$

$A-$-Average aspect ratio value

E-_-Average eccentricity

Table 3 Morphological feature parameter regression fitting

\begin{tabular}{lcccc}
\hline Regression fitting & Minimum value & Maximum & $\begin{array}{c}\text { Average } \\
\text { value }\end{array}$ & Variance \\
& & & 0.1070 & 0.0042 \\
Arthritis & 0.6341 & 1.0988 & -0.1867 & 0.0014 \\
Rice bacterial leaf streak & -0.2775 & -0.0642 & 0.7868 & 0.0120 \\
Chronic leaf blast & -0.0878 & 0.2108 &
\end{tabular}

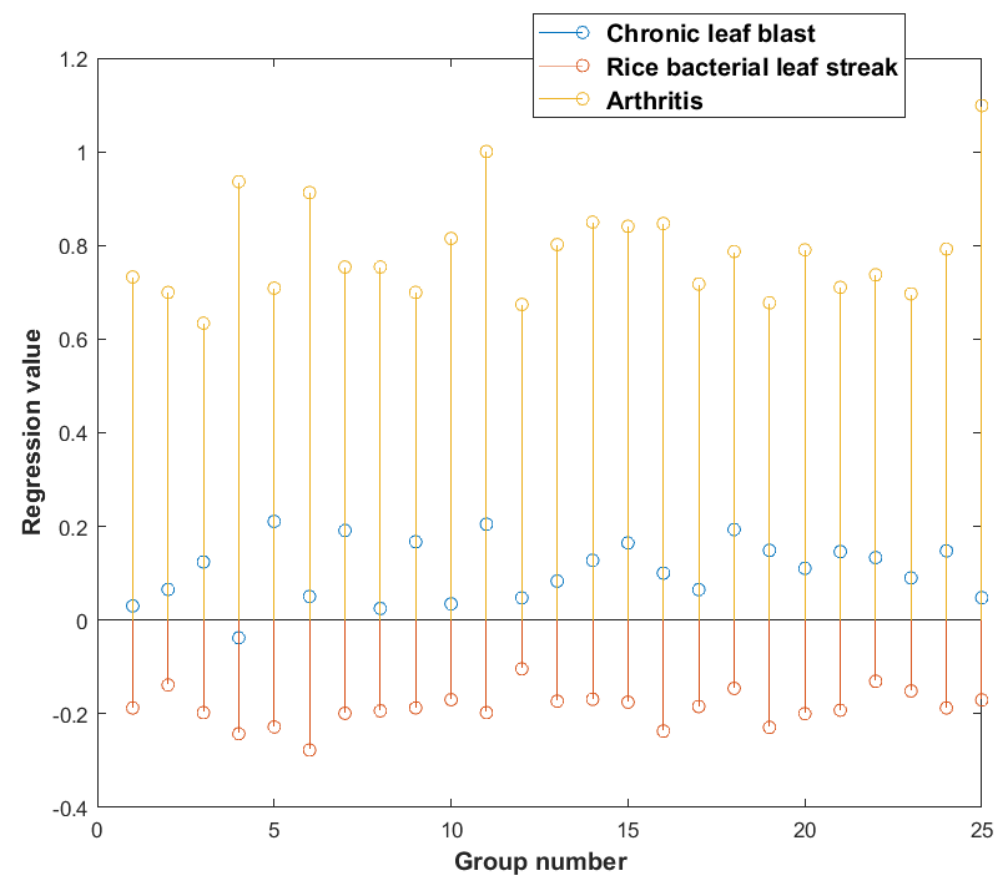

Figure 4: Binary regression values of morphological feature parameters

Figure 4 shows that the regression value of the knot plague is quite different from the those of the other two diseases. The average value of its morphological characteristics is approximately 0.78 , which can be more intuitive and accurate for identifying the disease.

The morphological characteristic values of the three diseases were used in the established binary regression prediction model for testing. The prediction model automatically converts the binary regression values obtained from the different groups into disease codes and then identifies and screens out the disease. Among them, 41 groups were verified as the festival plague, 38 groups were correctly identified, and the correct rate was $92.7 \%$.
Chronic leaf blast and bacterial leaf streak have cross sections and cannot be accurately distinguished.

Furthermore, binary regression fitting has a higher recognition rate for knots on the basis of a faster recognition rate.

\subsection{Step3BP Neural Network Model Prediction}

Chronic leaf blast and bacterial leaf streak have certain similarities in some aspects of the morphological characteristic values, and the recognition accuracy rate is low only through the binary linear regression equation model. 
In the third step, a BP neural network prediction model is established to predict and identify chronic leaf blast and bacterial leaf streak.

The BP neural network is composed of an input layer, a hidden layer, and an output layer. We established a $3 \times 6 \times 2$ three-layer BP network model, and the network uses the log-sigmoid transfer function.

$$
\begin{aligned}
& f(x)=\frac{1}{1+e^{x}} \\
& E=\frac{\sum_{i}\left(T_{i}+O_{i}\right)^{2}}{2}
\end{aligned}
$$

Ti-_Expected output

Oi-—Network output

\subsubsection{Input layer design}

For the input layer, three indicators, namely, the color feature, eccentricity, and aspect ratio, are designed to obtain the number of groups for the identification of chronic leaf blast and the bacterial stripe disease in the output layer. Therefore, the number of nodes selected by the input layer is three, and the number of nodes selected by the output layer is two.

\subsubsection{Intermediate hidden layer design}

In this BP neural network[23] design, the amount of data is small because the number of nodes in the input and output layers is relatively small. Considering that the number of hidden layer neurons is very large, the amount of calculation is large, thereby decreasing the recognition speed.
Therefore, the recognition accuracy should also be considered in setting the number of neurons. The number of neurons affects the expected error. No specific formula is used to determine the number of neurons in the hidden layer. Empirical formulas are generally used.

$$
l=\sqrt{n+m}+a
$$

$\mathrm{n}-$-Number of neurons in the input layer

$\mathrm{m}$--Number of neurons in the output layer

l-_This neural network chooses the number of hidden layer neurons to be 6

a-CConstant in $[1,10]$.

The network activation function is the tansigmoid function, the network output layer activation function is the log-sigmoid function, and the network performance function is mean squared error. The sample data are normalized and used in the network. Given the small amount of data, the number of training iterations (Epochs) is set to 500, the expected error value (Goal) to 0.001 , and the learning rate (Lr) to 0.01 . Each set of input layer has 50 data, including 30 in each chronic leaf blast and bacterial streak disease test group, and 20 for each verification group.

\subsection{Training Result}

Figure 5 shows that under the $3 \times 6 \times 1$ network model, the expected error value of 0.001 was reached after 185 iterations.

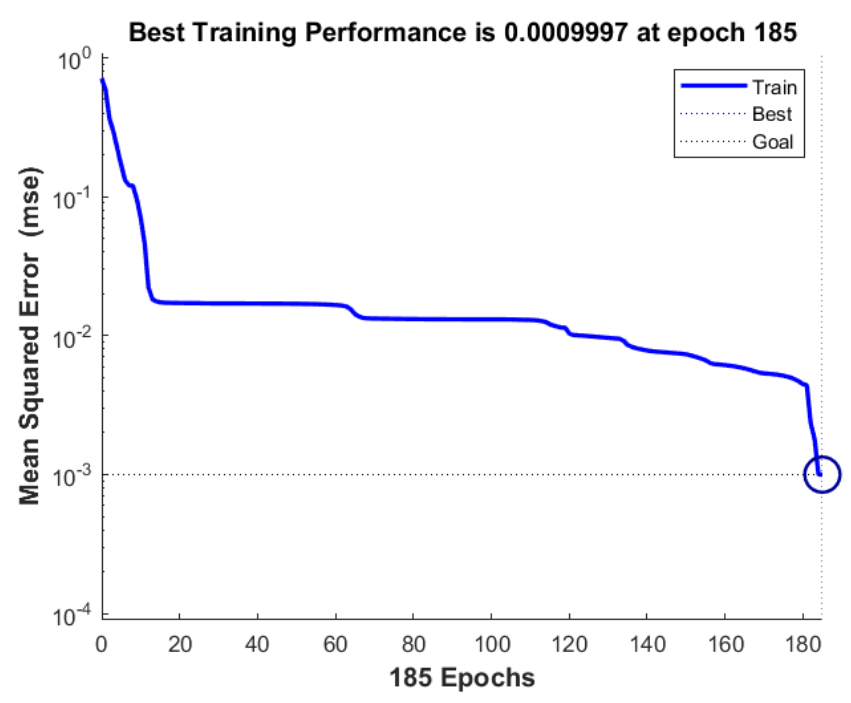

Figure 5: BP neural network training results

The BP neural network prediction model established using 150 sets of data is used to predict and identify diseases, verify 20 chronic leaf blast disease verification groups, and accurately predict 18 diseases, and the accuracy rate is $90 \%$. Then, 20 bacterial streak disease verification groups were verified, 19 were accurately predicted, and the accuracy rate is $95 \%$.

When the number of prediction groups is small, the prediction accuracy of BP neural network is 
relatively high, compensating for the low recognition rate of chronic leaf blast and linear streak disease in the binary linear regression model. Therefore, the binary regression model can only be used to screen out the knot blast disease, and the remaining chronic leaf blast and the relatively similar bacterial streak disease are identified by the BP neural network prediction model.

\subsection{CNN Disease Image Classification}

Based on the Deep Learning Toolbox provided by MATLAB2018a, a CNN was built in the Matlab environment for the secondary classification of diseased images because we binarized the RGB image in advance, segmented the lesion area, and artificially marked part of the lesion corresponding to the disease to reduce the amount of data processing. Therefore, we segmented the area into $28 \times 28$ parts, and the input layer built was $28 \times 28 \times$ 1.
The CNN we built is composed of a convolutional layer, a pooling layer, and a fully connected layer. Batch normalization layers were used in the convolutional layer and the nonlinear layer (ReLU layer) to optimize the problem and train faster. The convolutional layer uses convolution2Layer namevalue pair parameters to define the stride and learning rate of the layer. The downsampling operation is performed on the transformation to reduce the spatial size of the feature map and delete redundant spatial information. The maximum pooling layer returns the maximum value of the rectangular input area specified by the first parameter (poolSize). The OutputSize parameter in the last fully connected layer is equal to the number of classes in the target data, and the output here is 5 . The softmax layer is created after the last fully connected layer. The dropout function is added to prevent overfitting. The return value probability of the output layer determines whether to re-verify in the future.

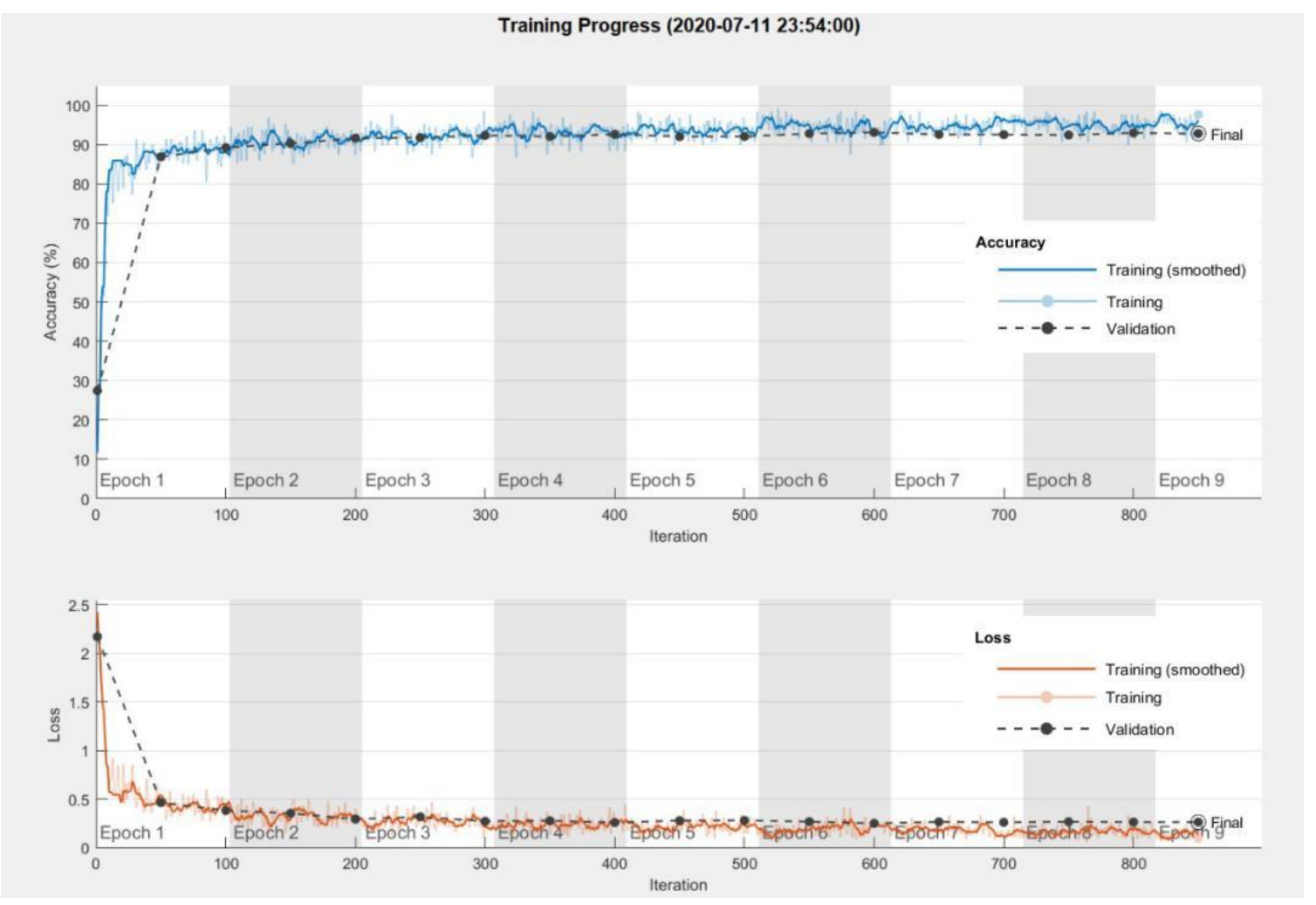

Figure 6: CNN training process

The CNN uses a sample size that is 0.5 times the training set, 0.3 times the validation set, and 0.2 the test set. For the validation set, the number of iterations is set to 30 , and the learning rate is 0.01 . The process can "stop early" after 9 iterations of Epoch, and training can be stopped if the 5 steps in the validation set loss are not reduced. The final training accuracy rate was $93.32 \%$, and the test set classification accuracy rate was $93.69 \%$.

\subsection{Experimental Verification}

To more intuitively determine the recognition effect of each step in the texture feature-multiple linear regression equation-BP neural network threestep recognition method, we split the three steps to test separately. Experiment was performed on 40 disease pictures in each group of 5 diseases that have been classified and processed. In the first step, leaf tip blight and healthy rice are studied to 
determine the accuracy and underreporting rate of the recognition by the texture feature value. From the pictures not recognized in Step 1, the knots to be are taken out. The pictures are included in the second step of the experiment. The pictures not recognized in Step 2 undergo three steps according to the above method. Each step records the recognition accuracy and false alarm rates of the target disease.

The average time is calculated by calculating the average time for each type of disease identification, and the result is kept to two decimal places.

Table 4 Disease identification results

\begin{tabular}{|c|c|c|c|c|c|c|c|c|}
\hline \multirow{2}{*}{$\begin{array}{c}\text { Serial } \\
\text { number }\end{array}$} & \multirow[b]{2}{*}{ Group } & \multirow{2}{*}{$\begin{array}{l}\text { recognition } \\
\text { methods }\end{array}$} & \multicolumn{2}{|c|}{ Number of samples } & \multirow{2}{*}{$\begin{array}{c}\text { Accuracy } \\
(\%)\end{array}$} & \multirow{2}{*}{$\begin{array}{c}\text { False } \\
\text { negative } \\
\text { rate } \\
(\%)\end{array}$} & \multirow{2}{*}{$\begin{array}{c}\text { False } \\
\text { alarm } \\
\text { rate } \\
(\%)\end{array}$} & \multirow{2}{*}{$\begin{array}{c}\text { time } \\
\text { consuming } \\
(\mathrm{s})\end{array}$} \\
\hline & & & $\begin{array}{l}\text { Number } \\
\text { of tests }\end{array}$ & $\begin{array}{c}\text { Actual } \\
\text { inspection } \\
\text { quantity }\end{array}$ & & & & \\
\hline \multirow{2}{*}{1} & \multirow{2}{*}{$\begin{array}{l}\text { Rice leaf } \\
\text { tip }\end{array}$} & A & 40 & $40 / 38$ & 95.0 & 5.0 & 5.0 & 4.65 \\
\hline & & B & 40 & $38 / 35$ & 92.1 & 12.5 & 7.5 & 3.17 \\
\hline \multirow{2}{*}{2} & \multirow{2}{*}{$\begin{array}{l}\text { Healthy } \\
\text { rice }\end{array}$} & A & 40 & $40 / 39$ & 97.5 & 2.5 & 2.5 & 4.75 \\
\hline & & B & 40 & $40 / 38$ & 95.0 & 5.0 & 5.0 & 3.13 \\
\hline \multirow{3}{*}{3} & \multirow{3}{*}{ Arthritis } & A & 40 & $40 / 37$ & 92.5 & 7.5 & 7.5 & 4.77 \\
\hline & & & & & & & & \\
\hline & & B & 40 & $41 / 36$ & 87.8 & 10.0 & 12.5 & 3.74 \\
\hline \multirow{2}{*}{4} & Chronic & A & 40 & $40 / 37$ & 92.5 & 7.5 & 7.5 & 4.98 \\
\hline & blast & B & 40 & $43 / 39$ & 90.7 & 2.5 & 10.0 & 4.32 \\
\hline \multirow{2}{*}{5} & \multirow{2}{*}{$\begin{array}{l}\text { Bacterial } \\
\text { streak }\end{array}$} & A & 40 & $40 / 40$ & 100.0 & 0 & 0 & 4.63 \\
\hline & & B & 40 & $36 / 35$ & 97.2 & 12.5 & 2.5 & 4.29 \\
\hline
\end{tabular}

In Table 4, the CNN is used as the first step to identify, and the method of re-verification of the 3step recognition method is recorded as $A$; the method of only using the 3-step recognition method is recorded as B.

\subsection{Result Analysis}

The recognition process is divided into three steps, and recognition is performed layer by layer by gradually adding recognition conditions. The texture feature (the ratio of the gray-white area in the center of the disease spot to the entire disease spot area) is added in Step 1, and the healthy rice and leaf blight among the five diseases are identified and screened.

The texture characteristics of leaf blight and healthy rice are obviously different. The texture feature can be used again for secondary screening to identify leaf tip blight and healthy rice; the recognition rate of healthy rice is $100 \%$. In Step 2, the eccentricity and aspect ratio of the morphological feature values are added to establish a binary regression model.

The knot plague can be quickly detected and identified because of the small amount of calculation of the fitting regression model. The recognition accuracy rate is $92.7 \%$, but a $7.5 \%$ underreport is observed. These underreporting groups directly affect the number of samples tested in the third step. In Step 3, the color feature value is added again, and the morphological feature value constitutes the input layer of the BP neural network prediction model.

The BP model predicts and recognizes the remaining two similar diseases. The 3 -step screening and recognition model constructed in this study has an average recognition accuracy of $92.4 \%$. The softmax activation function is used in the output layer of the CNN to classify the probability of each input return, and the group with a low probability is again subjected to the 3-step recognition for verification. Its comprehensive recognition accuracy rate is $95.5 \%$. 


\section{Disease Level Detection}

\subsection{Evaluation Criteria for Rice Disease Resistance}

Table 5 Rice blast disease levels and resistance evaluation criteria

\begin{tabular}{lll}
\hline Disease level & Resistance level & Incidence \\
\hline 0 & HR & Disease-free \\
1 & MR & Small gray lesions, diameter less than $1 \mathrm{~mm}$ \\
2 & R & Small gray lesions, about $1-2 \mathrm{~mm}$ in diameter \\
3 & MS1 & $\begin{array}{l}\text { Typical spindle lesions, accounting for less than } 2 \% \text { of } \\
\text { the leaf area }\end{array}$ \\
4 & MS2 & Typical lesions, affected area $2.1 \%-10 \%$ \\
5 & S1 & Typical lesions, affected area $10.1 \%-25 \%$ \\
6 & S2 & Typical lesions, affected area $25.1 \%-50 \%$ \\
7 & HS1 & Typical lesions, affected area $50.1 \%-75 \%$ \\
8 & HS2 & All the leaves die
\end{tabular}

\subsection{Disease Classification Process}

To verify the accuracy of the experiments using the above calculation methods and grading standards, two other methods were adopted, namely, manual visual inspection and the Photoshop software, to manually segment the collected images to calculate the lesion areas. Finally, grading was performed according to the ratio of the area of the diseased spot to the area of the leaf[24]. The number of experimental samples is 30 , and the experimental results are shown in Table 6.

Table 6 Three methods to classify different leaves

\begin{tabular}{|c|c|c|c|c|c|}
\hline \multirow[t]{2}{*}{ Group } & \multicolumn{3}{|c|}{ Detection method } & \multirow{2}{*}{$\begin{array}{c}\text { Visual } \\
\text { accuracy } \\
(\%)\end{array}$} & \multirow{2}{*}{$\begin{array}{c}\text { The accuracy } \\
\text { of this method } \\
(\%)\end{array}$} \\
\hline & Photoshop & $\begin{array}{l}\text { This } \\
\text { method }\end{array}$ & $\begin{array}{c}\text { Visual } \\
\text { inspection }\end{array}$ & & \\
\hline Chronic leaf blast & 30 & 28 & 25 & 83.3 & 93.3 \\
\hline $\begin{array}{l}\text { Rice bacterial leaf } \\
\text { streak }\end{array}$ & 30 & 27 & 24 & 80.0 & 90.0 \\
\hline Rice leaf tip blight & 30 & 25 & 25 & 83.3 & 83.3 \\
\hline
\end{tabular}

The accuracy of this experimental method and the Photoshop classification result exceeds $80 \%$. The experimental method has a higher recognition accuracy than the manual visual inspection method and can replace the human visual inspection method.

\section{Disease Identification Application}

To realize the preliminary application of disease identification, this study built an application development environment through Android Studio 3.4.2 under the Windows 10 operating system.
The development environment uses various software development kits, including the Android Software Development Kit, the Java Development Kit, Android Development Tools, and OpenCV 3.4.3. According to the parameters obtained in MATLAB2018a, an application software that can process pictures and detect and classify diseases on mobile phones was written.

The application can use mobile phones to take pictures and obtain images from the gallery and call the OpenCV database to perform image processing and feature extraction on the obtained images of rice leaves. 

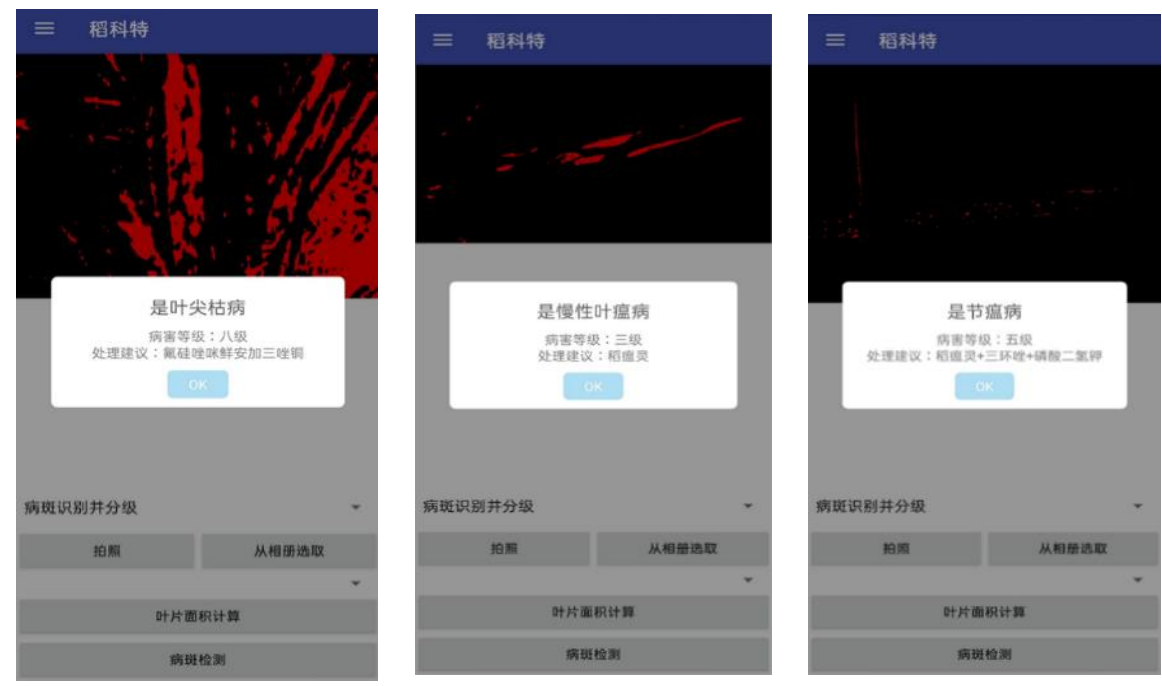

Figure 7: Mobile application recognition example

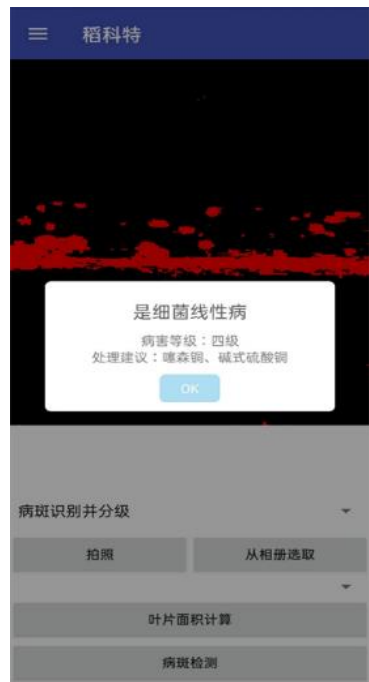

[2] Lai J C, Li S K, Ming B,et al. Advances in research on computer-vision diagnosis of crop diseases. Scientia Agricultura Sinica, 2009, 42(4): 12151221.

[3] Chen B Q, Guo X M, Li X H. Image diagnosis algorithm of diseasedwheat[J].Transactions of the Chinese Society for Agricultural Machinery, 2009, 42(12): 190-195.

[4] Wen C J, Wang S S, Yu H L, et al.Mage segmentation method for maize diseases based on pulse coupled neural network with modified artificial bee algorithm[J].Transactions of the Chinese Society of Agricultural Engineering, 2013, 29(13): 142-149.

[5] Wang N, Wang $\mathrm{K} \mathrm{R}, \mathrm{T}$, Xie $\mathrm{R}$ Z,et al. Image recognition of corn leaf diseases based on Fisher discriminant analysis [J] .Chinese Journal of Agricultural Sciences, 2009, 42 (11): 3836-3842. (in Chinese)

[6] Zhou Z, Lei M L, Tang S X. Study of tomato leaf disease recognition based on computer vision technology[J]. Journal of Anhui Agricultural Sciences, 2013, 41(5): 2288-2289.

[7] Zhang J H, Qi L J, Ji R H,et al. Cotton diseases identification based on rough sets and BP neural network[J]. Transactions of the Chinese Society of Agricultural Engineering, 2012, 28(7): 161-167.

[8] Zhang Y J.Image engineering-Image analysis[M].Beijing, Tsinghua University Press,2005,73-105.

[9] Li J P, Zhu W H. Occurrence and control of bacterial brown streak in rice[J]. Guizhou Agricultural Sciences, 2003, 31(2): 34-36.

[10] Qiu J,Liu J R,Cao Z Y,et al.Rice Disease Image Recognition Research Based on Convolutional Neural Network [J]. Journal of Yunnan Agricultural University (Natural Science),2019,34(5):884-888DOI:10.12101/j.iss n.1004-390X(n).201805010. 
[11] Tan Y L,Ouyang C J,Li L,et al. IMAGE RECOGNITION OF RICE DISEASES BASED ON DEEP CONVOLUTIONAL NEURAL NETWORK[J].Journal of Jinggangshan University (Natural Science) ,2019, 40 (2) : 31-38.

[12] Chen F R.Rice Diseases and Insect Pests Atlas and Control Technology.Beijing:China Agriculture Science Press, 2012.

[13] Xia S G,Tang Q Y.Rice Disease Pest and Weed Prevention Atlas.Beijing:China Agriculture Press, 2006.

[14] M JJones,J M Rehg.Statistical color models with application to skin detection[J].Int' Journal of Computer Vision archive,2002,46:81-96.

[15] N.Oliver,A.Pentland,F.Berard.LAFTER:A realtime face lips tracker with facial expression recognition[J].Pattern Recognition,2000,33:13691382.

[16] Hu C.Qin S H.Design of license plate positioning system based on color texture [J]. Journal of Wuhan University Engineering,2017,39(3) : 273-280.

[17] Dou J J,Wen J,Liu C Q.Histogram-based color image retrieval [J]. Infrared and Laser Engineering, 2005, 34(1) : 84-88.
[18] Huang Z K. A study of feature extraction technique based on color image and plant classification[D]. Hefei: University of Science and Technology of China, 2006.

[19] Long W F.Rice seedling blight and downy mildew identification and control techniques[J].Plant Protection Technology and Extension, 2003, 23(4): 34-35.

[20] Wang X M, Wang T X, Wu M X. Downy mildew and red rice blight identification and prevention measures. Plant Doctor, 2011, 24(2): 4-5.

[21] Li J P, Zhu W H. Occurrence and control of bacterial brown streak in rice. Guizhou Agricultural Sciences[J], 2003, 31(2): 34-36.

[22] Lai C Y, Yuan G Q. Agricultural Phytopathology(Southern). Beijing: Science Press, 2008. (in Chinese)

[23] Werbos P.J.The roots of backpropagation:from ordered derivatives to neural networks and political forecasting.New York, USA: John Wiley, 1994.

[24] Guan Z X,Tang J.Y B J,et al.Research on Rice Disease Recognition Method Based on Image [J]. Chin J Rice Sci,2010,24(5):497 502DOI:10.3969/j.issn.1001 -7216.2010 .05 .009 . 